\title{
Gut Microbiota Changes During Helicobacter Pylori Eradication with Vonoprazan-Containing Triple Therapy Among Adolescents: A Prospective Multicenter Study in Japan
}

Toshihiko kakiuchi ( $\nabla$ kakiucht@cc.saga-u.ac.jp )

Saga University Hospital https://orcid.org/0000-0002-9995-5522

Kentaroh Yamamoto

Yamamoto Memorial Hospital

Ichiro Imamura

Imamura Hospital

Kazutoshi Hashiguchi

Imamura Hospital

Hiroharu Kawakubo

Imariarita Hospital

Daisuke Yamaguchi

Ureshino Medical Center

Yasuhiko Fujioka

Fujioka Hospital

Aiko Nakayama

Saga Daigaku Igakubu Fuzoku Byoin

Masumi Okuda

Hyogo Ika Daigaku Byoin

Research article

Keywords: Actinobacteria, Feces, Gastrointestinal Microbiome, Adolescent, Body Fluids

Posted Date: June 24th, 2020

DOI: https://doi.org/10.21203/rs.3.rs-37210/v1

License: (c) (1) This work is licensed under a Creative Commons Attribution 4.0 International License.

Read Full License 


\section{Abstract}

Background: Whether the screening and treatment of Helicobacter pylori infection are safe for children is debated in Japan. This study aimed to evaluate the safety of Helicobacter pylori eradication therapy by examining long-term changes in the gut microbiota during therapy among children.

Methods: Children with anti-Helicobacter pylori antibody in the urine and Helicobacter pylori antigen in the feces were enrolled in this study. Their stool samples were collected at three time points, which were as follows: prior to treatment, 1-2 days after treatment, and time of judgment of eradication therapy. After treatment, the relative abundance, alpha-diversity, and beta-diversity of the gut microbiota were assessed.

Results: In 16 students finally included in the study, the number of Actinobacteria isolated decreased immediately after eradication therapy, and it returned to pretreatment condition at the judgment point. There was no change at the genus level. The alpha-diversity was lost immediately after eradication therapy. However, it recovered at the time of eradication judgment, and it was restored before eradication therapy. Meanwhile, there was no change in beta-diversity, and none of the participants experienced serious adverse events.

Conclusion: Helicobacter pylori eradication therapy is safe even for children in the view point of gut microbiota. Thus, further long-term evaluations of changes in the gut microbiota after eradication therapy and an assessment of healthy children without Helicobacter pylori must be performed. These will in turn promote screening and treatment among adolescents to prevent gastric cancer.

Trial registration: This study was registered with the University Hospital Medical Information Network Clinical Trials Registry (no. UMIN000028726, https://upload.umin.ac.jp/cgi-bin/ctr_e/ctr_view.cgi? recptno=R000032873) on 18 August 2017.

\section{Background}

Helicobacter pylori ( $H$. pylori) infection is generally established in the family at $\leq 5$ years of age in Japanese children [1], and it causes atrophic gastritis in childhood [2-5]. H. pylori eradication therapy is effective if initiated immediately after infection, and it causes minimal damage to the gastric mucosa [6]. Furthermore, it can reduce the risk of developing next-generation gastric cancer [7]. Considering that the current infection route of $H$. pylori is person-to-person transmission, particularly within the same family [1], [8], young individuals must take measures against $H$. pylori infection from the viewpoint of preventing this infection in the next-generation. Based on such circumstances, as a new method of preventing gastric cancer, the screening and treatment of $H$. pylori infection among young individuals have begun as a primary prevention in Japan [9], [10], [11], [12].

In Japan, triple therapy with potassium-competitive acid blocker ( $\mathrm{P}-\mathrm{CAB}$ ), amoxicillin, and clarithromycin is an effective first-line treatment for $H$. pylori infection in adults $[13,14]$ and children $[9,12]$. To date, $H$. pylori eradication therapy has no serious side effects in children [9], [15]. However, a long-term evaluation 
was not conducted. Moreover, individuals without $H$. pylori infection present with few allergic diseases [16]. To date, the safety of $H$. pylori eradication therapy using proton-pump inhibitor and P-CAB in children is controversial. A previous study reported the effect of probiotics during vonoprazan-containing triple therapy on the intestinal microbiota in individuals with H. pylori infection [17]. However, only the intestinal microbiota before and immediately after eradication therapy were evaluated. Thus, this study aimed to evaluate the safety of $H$. pylori eradication therapy by examining the long-term changes in the gut microbiota during therapy among children.

\section{Methods}

\section{Study design and participants}

This study conducted a sub-analysis of $H$. pylori infection screening as part of the junior high school health screening system among third-year students in Saga Prefecture [9]. Using local governmental grants, a screening and treatment program for eradicating $H$. pylori infection among third-grade junior high students was started in Saga Prefecture in 2016. The students underwent urinary anti- $H$. pylori antibody tests (RAPIRAN; Otsuka Pharmaceutical Co., Ltd. Tokyo, Japan), followed by H. pylori stool antigen tests (TESTMATE RAPID RYLORI ANTIGEN; Wakamoto Pharmaceutical Co., Ltd. Tokyo, Japan). To eradicate $H$. pylori infection, those who tested positive on both tests received triple therapy comprising 20 mg vonoprazan (Takeda Pharmaceutical Co., Ltd. Tokyo, Japan), $750 \mathrm{mg}$ amoxicillin, and $200 \mathrm{mg}$ clarithromycin twice a day for 7 days. Then, 8-12 weeks after the eradication therapy, the ${ }^{13} \mathrm{C}$-urea breath test was conducted at one of the cooperating medical institutions to assess for treatment efficacy. Breath samples were obtained $4 \mathrm{~h}$ after a meal and $20 \mathrm{~min}$ after the ingestion of $100 \mathrm{mg}{ }^{13} \mathrm{C}$-urea (UBIT ${ }^{\circledR}$ tablet, $100 \mathrm{mg}$ ). An infrared spectrometer (POC One ${ }^{\circledR}$; Otsuka Electronics Co., Ltd., Hirakata, Japan) was used for the test.

Children with two positive test results were enrolled in the study between June 2017 and March 2019. Stool samples were collected prior to treatment (Before), 1-2 days after treatment (After), and after 8-12 weeks of eradication therapy (Judgment). At the time of judgment of eradication therapy, students with unsuccessful treatment were excluded to rule out the effect of the presence of $H$. pylorion the gut microbiota [18]. The outcome of $H$. pylori eradication therapy was confirmed using the ${ }^{13} \mathrm{C}-\mathrm{UBT}$. The stools were immediately stored at $-20^{\circ} \mathrm{C}$ until DNA extraction.

This study was approved by the ethics committee of Saga University (institutional review board no.: 2017-03-01) and was registered in the clinical trials registry (UMIN000028726) of the University Hospital Medical Information Network. All patients and their parents provided a written informed consent for participating in the study and for collecting fecal samples.

\section{DNA extraction}


The specimens were stored at $-20^{\circ} \mathrm{C}$ until DNA extraction. Bacterial DNA was extracted using the NucleoSpin Microbial DNA kit (MACHERY-NAGEL, Düren, Germany) according to the manufacturer's instructions. The total DNA was eluted in $50 \mu \mathrm{L}$ of elution buffer and was stored at $-20^{\circ} \mathrm{C}$. The $\mathrm{V} 3-\mathrm{V} 4$ hypervariable regions of $16 \mathrm{~S}$ ribosomal DNA (rDNA) were amplified using the $16 \mathrm{~S}$ (V3-V4) Metagenomic Library Construction Kit for NGS (Takara Bio Inc., Kusatsu, Japan) with primer pairs (341F 5'TCGTCGGCAGCGTCAGATGTGTATAAGAGACAGCCTACGGGNGGCWGCAG-3', 806R 5' GTCTCGTGGGCTCGGAGATGTGTATAAGAGACAGGGACTACHVGGGTWTCTAAT-3'). The amplicon was purified using AMPure XP magnetic beads (Beckman Coulter, Brea, CA, the USA). The index PCR was performed using the Nextera XT Index Kit (Illumina, San Diego, CA, the USA). After purification with AMPure XP beads, sequencing was conducted on a MiSeq platform with the MiSeq Reagent Kit v3 and Phix Control Kit v3 (Illumina) from Takara Bio Inc.

\section{16s rDNA sequence analysis}

The 16s rDNA sequencing results were analyzed as follows: low-quality sequences were removed, chimeras were checked, operational taxonomic units (OTUs) were constructed, and taxonomy was assigned using CD-HIT-OTU, Quantitative Insights Into Microbial Ecology pipeline (http://qiime.org/). OTUs were established by clustering with a $97 \%$ identity threshold and were completed using an RDP classifier with the Green genes database. The observed OTUs, Chao1 (microbial species richness), and Shannon indices (microbial evenness) and PD whole tree (phylogenetic richness) were calculated to determine the alpha (a)-diversity of the microbiota in the samples. The beta ( $\beta$ )-diversity of our samples was calculated using the default beta-diversity metrics of the weighted and unweighted UniFrac distance. To compare the differences in the overall bacterial gut microbiota structure, a principal coordinates analysis was performed to reduce the dimensionality of the resulting distance.

\section{Statistical analysis}

The raw data were expressed as percentage, mean, and standard deviation whenever applicable. For skewed data, the Mann-Whitney $U$ test and Wilcoxon signed-rank test were used to compare variables between and within groups, respectively. The comparison of a-diversity and group distance for $\beta$-diversity were performed using the Monte Carlo two-sample test, followed by the false discovery rate and Bonferroni correction. A $P$ value $<0.05$ was considered statistically significant. Statistical analyses of the microbiota were performed using Python. Other statistical analyses were performed using R ( $\mathrm{R}$ Core Team 2018]. R: A language and environment for statistical computing; R Foundation for Statistical Computing, Vienna, Austria; URL: https://www.R-project.org/.).

\section{Study endpoint}

The study endpoint was the change in the intestinal microbiota during $H$. pylori eradication with vonoprazan-containing triple therapy. In particular, the relative abundance, $\alpha$-diversity, and $\beta$-diversity of the gut microbiota were compared before eradication, immediately after eradication, and during 
assessment of treatment efficacy. In addition, the adverse effects of H. pylori eradication with vonoprazan-containing triple therapy in children were evaluated.

\section{Results}

The flow of patient enrolment is presented in Figure 1. Of the 150 patients with H. pylori infection, 31 provided consent and participated in this study. There were 29 students who provided stool samples before eradication, 26 immediately after eradication, and 19 at the time of eradication judgment. Of the 19 patients who provided stool samples at the time of the eradication judgment, three experienced primary treatment failure. Finally, $16(n=9$, male participants, $n=7$, female participants; average age: 15.5 years [standard deviation: 0.34 years]) were eligible for this study. Then, these patients submitted the stool samples at each of the three time points. The $H$. pylori eradication assessments were conducted on the 89th median day after the eradication therapy, and the stool samples were submitted on the 95th median day.

\section{Relative abundance}

Figure 2 shows the compositions and relative abundance of the gut microbiota at the phyla level. The proportion of Actinobacteria significantly decreased after eradication therapy compared with before eradication therapy $(P=0.006924)$. On the contrary, the proportion of Actinobacteria significantly increased at the time of eradication assessment compared with after eradication therapy $(P=0.047065)$, and no significant difference was observed between before eradication therapy and at the time of eradication assessment $(P=0.214554)$. Figure 3 shows the top seven genus compositions of the relative abundance of gut microbiota. In each of the seven genus, no significant changes were observed in the comparison results before eradication therapy, after eradication therapy, and during eradication judgment.

\section{Alpha-diversity}

We calculated the a-diversity according to the PD whole tree, Shannon index, observed OTUs, and Chao1 at three points. We found significantly lower phylogenetic richness (PD whole tree; $P=0.003$ ), evenness of microbiota (Shannon index; $P=0.023$ ), and microbial species richness (observed OTUs; $P=0.003$, Chao1; $P=0.001)$ after eradication therapy compared with before eradication therapy. However, there were no significant differences in terms of the PD whole tree $(P=1.000)$, Shannon index $(P=1.000)$, observed OTUs $(P=1.000)$, and Chao1 $(P=1.000)$ at the time of eradication judgment compared with before eradication therapy. Moreover, the PD whole tree $(P=0.063)$, Shannon index $(P=0.492)$, observed OTUs $(P=0.106)$, and Chao1 $(P=0.061)$ did not significantly differ at eradication judgment point compared with after eradication therapy (Fig. 4).

\section{Beta-diversity}


The $\beta$-diversity, calculated based on the weight and unweight UniFrac distance, revealed that the gut microbiota structures were between before and after eradication therapy (Weight UniFrac distance: Before-Before vs. Before-After, $P=0.03$; After-After vs. Before-After, $P=1.00$. Unweight UniFrac distance: Before-Before vs. Before-After, $P=0.03$; After-After vs. Before-After, $P=0.98$ ) (Fig. 5). Similarly, the $\beta$-diversity revealed that the gut microbiota structures were between before eradication therapy and judgment of eradication therapy (Weighted UniFrac distances: Before-Before vs. BeforeJudgment, $P=1.00$; Judgment-Judgment vs. Before-Judgment, $P=1.00$. Unweighted UniFrac distances; Before-Before vs. Before-Judgment, $P=1.00$; Judgment-Judgment vs. Before-Judgment, $P$ $=0.87$ ) (Fig. 6).

\section{Adverse events}

In total, $1(6.35 \%)$ of patients presented with diarrhea and $2(12.5 \%)$ of 16 with abdominal pain. None of the adverse events were serious. Thus, therapy was not required, and treatment for the eradication of $H$. pylori was not interrupted.

\section{Discussion}

This study showed changes in the gut microbiota before and after $H$. pylori eradication therapy using next-generation sequencing and amplicon analysis in Japanese children. Two important clinical suggestions were obtained. First, change in the gut microbiota during eradication therapy of $H$. pylori with vonoprazan-containing triple therapy in children caused dysbiosis immediately after eradication therapy. However, it returned to pretreatment conditions 3 months after the eradication therapy. Second, the eradication therapy of $H$. pylori with vonoprazan-containing triple therapy was found to be safe for children.

Yap TW et al. reported that the eradication of H. pylori caused perturbation of the gut microbiota, and it may indirectly affect the health of humans [19]. By contrast, Gotoda T et al. reported that although $H$. pylori eradication therapy caused short-term dysbiosis, microbial diversity was restored in healthy Japanese teenagers [20]. This is the first study that evaluated the changes in the gut microbiota before and after $\mathrm{H}$. pylori eradication therapy in adolescents. Moreover, a-diversity analysis revealed that both species microbial richness and evenness were recovered to per-treatment levels at 2 months after eradication therapy, and the proportion of Actinobacteria significantly decreased immediately after eradication therapy, and it recovered similarly after 2 months of post-eradication. Our results were quite similar to those of the previous study. Liou JM et al. reported that although the eradication therapy of $H$. pylori infection has minimal disruption in the microbiota, their results support the long-term safety of $\mathrm{H}$. pylori eradication therapy [21]. Because dysbiosis of the gut microbiota was recovered after several months before initiation of the eradication therapy of H. pylori, the safety of eradication therapy among children may be recommended from the viewpoint of the gut microbiota. Furthermore, the adverse events observed in our participants were diarrhea and abdominal pain. However, these side effects were not serious. 
Several studies showed that $H$. pylori infection in children caused the changes in the gut microbiota. In adults with $H$. pylori infection, the intestinal microbiota has reduced diversity [22]. Benavides-Ward et al. reported that children with $H$. pylori infection increased the number of bacteria, including Proteobacteria, Clostridium, Firmicutes, and Prevotella, in the gut microbiota [18]. However, information regarding the relationship between $H$. pylori infection and intestinal bacteria from the microbiota is still limited. Since there are differences in gut microbiota between $H$. pylori-infected and non-infected children, $H$. pylori must be eradicated. However, future studies must be conducted to compare the gut microbiota between children who have passed for a long time after $H$. pylori eradication therapy and healthy children without H. pylori infection.

In Japan, the screening and treatment of H. pylori infection among adolescents are performed as a primary preventive measure against gastric cancer. By contrast, the clinical practice guidelines do not recommend screening and treatment for asymptomatic $H$. pylori-infected children to prevent gastric cancer [23], [24]. However, these guidelines are for children and adolescents living in Europe and North America, and they may not be applicable to those living in other continents, particularly in developing countries with a high $H$. pylori infection rate and limited health care resources [25]. The safety of $H$. pylori screening and treatment for children is debated in Japan. Our study showed that dysbiosis developed immediately after $\mathrm{H}$. pylori eradication therapy. However, it returned to pretreatment condition after 3 months. This may be one factor for the safety of $H$. pylori eradication treatment. To prevent gastric cancer, $H$. pylori infection must be eradicated at a young age [6]. To Moreover, the safety of $H$. pylori eradication therapy for children must be further validated [26], [7].

This study had several limitations. First, the sample size was small. Second, this study did not include placebo treatment for comparison. Third, the altered intestinal flora could be evaluated, but the functionality of the bacteria itself and how it affected humans were not evaluated.

\section{Conclusion}

H. pylori eradication therapy is safe even for children in the view point of gut microbiota. Thus, even further long-term evaluations of the changes in the gut microbiota after eradication therapy and an assessment of healthy children without $H$. pylori infection must be performed. These will in turn promote screening and treatment among adolescents to prevent gastric cancer.

\section{Abbreviations}

Helicobacter pylori

H. pylori; potassium-competitive acid blocker:P-CAB; ribosomal DNA:rDNA; operational taxonomic units:OTUs; alpha:a; beta: $\beta$;

\section{Declarations}




\section{Ethics approval and consent to participate}

The institutional review boards of Saga University Hospital (approval numbers: 2017-03-01) approved the present study. This study was registered with the University Hospital Medical Information Network Clinical Trials Registry (no. UMIN000028726, https://upload.umin.ac.jp/cgi-bin/ctr_e/ctr_view.cgi? recptno=R000032873) on 18 August 2017.

Written informed consent was obtained from all participants and their parents or guardians.

\section{Consent for publication}

Written informed consents were obtained from all patients for publication of this manuscript.

\section{Availability of data and materials}

The datasets used and/or analyzed during the current study are available from the corresponding author on reasonable request.

\section{Competing interests}

TK received research funding from Biofermin Pharmaceutical Co., Ltd. The other authors declare no conflicts of interest.

\section{Funding information}

This study was supported by the Biofermin Pharmaceutical Co., Ltd.

\section{Authors' contributions}

TK and MO conceptualized, analyzed data, and prepared the manuscript; TK, AM, KY, II, KH, HK, DY, YF and AN collected data; $\mathrm{MO}$ critically reviewed the manuscript. All authors approved the final version of the article, including the authorship list.

\section{Acknowledgments}

We would also like to thank the outpatient nurses and medical support staff at the joint research institutes. We are also grateful to Ms. Kozue kakiuchi, Ms. Tomomi Ito, and Ms. Hiromi Beppu for providing support for the project. The Biofermin Pharmaceutical Co., Ltd., provided support for this study. However, this company was not involved in the analysis or interpretation of the test results.

\section{References}

1. Konno M, Fujii N, Yokota S, Sato K, Takahashi M, Sato K, Mino E, Sugiyama T. Five-year follow-up study of mother-to-child transmission of Helicobacter pylori infection detected by a random amplified polymorphic DNA fingerprinting method. J Clin Microbiol. 2005;43(5):2246-50. 
2. Ozawa K, Kato S, Sekine H, Koike T, Minoura T, linuma K, Nagura H. Gastric epithelial cell turnover and mucosal protection in Japanese children with Helicobacter pylori infection. Journal of gastroenterology. 2005;40(3):236-46.

3. Brigic E, Hadzic D, Mladina N. Childhood and Coress model of carcinogenesis. Med Arch. 2012;66(6):375-7.

4. Boukthir S, Aouididi F, Mazigh Mrad S, Fetni I, Bouyahya O, Gharsallah L, Sammoud A. [Chronic gastritis in children]. Tunis Med. 2007;85(9):756-60.

5. Yu Y, Su L, Wang X, Wang X, Xu C. Association between Helicobacter pylori infection and pathological changes in the gastric mucosa in Chinese children. Intern Med (Tokyo Japan). 2014;53(2):83-8.

6. Asaka M. A new approach for elimination of gastric cancer deaths in Japan. Int J Cancer. 2013;132(6):1272-6.

7. Kato M, Ota H, Okuda M, Kikuchi S, Satoh K, Shimoyama T, Suzuki H, Handa O, Furuta T, Mabe K, et al. Guidelines for the management of Helicobacter pylori infection in Japan: 2016 Revised Edition. Helicobacter. 2019;24(4):e12597.

8. Yokota S, Konno M, Fujiwara S, Toita N, Takahashi M, Yamamoto S, Ogasawara N, Shiraishi T. Intrafamilial, Preferentially Mother-to-Child and Intraspousal, Helicobacter pylori Infection in Japan Determined by Mutilocus Sequence Typing and Random Amplified Polymorphic DNA Fingerprinting. Helicobacter. 2015;20(5):334-42.

9. Kakiuchi T, Matsuo M, Endo H, Nakayama A, Sato K, Takamori A, Sasaki K, Takasaki M, Hara M, Sakata Y, et al. A Helicobacter pylori screening and treatment program to eliminate gastric cancer among junior high school students in Saga Prefecture: a preliminary report. Journal of gastroenterology. 2019;54(8):699-707.

10. Kusano C, Gotoda T, Ishikawa H, Moriyama M. The administrative project of Helicobacter pylori infection screening among junior high school students in an area of Japan with a high incidence of gastric cancer. Gastric Cancer. 2017;20(Suppl 1):16-9.

11. Akamatsu T, Ichikawa S, Okudaira S, Yokosawa S, Iwaya Y, Suga T, Ota H, Tanaka E. Introduction of an examination and treatment for Helicobacter pylori infection in high school health screening. Journal of gastroenterology. 2011;46(12):1353-60.

12. Kaji E, Yoden A, Otani M, Okuhira T, Aomatsu T, Tamai H, Ashida A. Helicobacter pylori test-and-treat strategy for second-year junior high school students aimed at the prevention of gastric cancer in Takatsuki City. Helicobacter 2020:e12696.

13. Murakami K, Sakurai Y, Shiino M, Funao N, Nishimura A, Asaka M. Vonoprazan, a novel potassiumcompetitive acid blocker, as a component of first-line and second-line triple therapy for Helicobacter pylori eradication: a phase III, randomised, double-blind study. Gut. 2016;65(9):1439-46.

14. Suzuki S, Gotoda T, Kusano C, Iwatsuka K, Moriyama M. The Efficacy and Tolerability of a Triple Therapy Containing a Potassium-Competitive Acid Blocker Compared With a 7-Day PPI-Based LowDose Clarithromycin Triple Therapy. Am J Gastroenterol. 2016;111(7):949-56. 
15. Okuda M, Kikuchi S, Mabe K, Osaki T, Kamiya S, Fukuda Y, Kato M. Nationwide survey of Helicobacter pylori treatment for children and adolescents in Japan. Pediatr Int. 2017;59(1):57-61.

16. Lionetti E, Leonardi S, Lanzafame A, Garozzo MT, Filippelli M, Tomarchio S, Ferrara V, Salpietro C, Pulvirenti A, Francavilla R, et al. Helicobacter pylori infection and atopic diseases: is there a relationship? A systematic review and meta-analysis. World J Gastroenterol. 2014;20(46):17635-47.

17. Kakiuchi T, Mizoe A, Yamamoto K, Imamura I, Hashiguchi K, Kawakubo H, Yamaguchi D, Fujioka Y, Nakayama A, Okuda M, et al: Effect of probiotics during vonoprazan-containing triple therapy on gut microbiota in Helicobacter pylori infection: A randomized controlled trial. Helicobacter 2020:e12690.

18. Benavides-Ward A, Vasquez-Achaya F, Silva-Caso W, Aguilar-Luis MA, Mazulis F, Urteaga N, Del ValleMendoza J: Helicobacter pylori and its relationship with variations of gut microbiota in asymptomatic children between 6 and 12 years. BMC research notes 2018, 11(1):468.

19. Yap TW, Gan HM, Lee YP, Leow AH, Azmi AN, Francois F, Perez-Perez GI, Loke MF, Goh KL, Vadivelu J. Helicobacter pylori Eradication Causes Perturbation of the Human Gut Microbiome in Young Adults. PloS one. 2016;11(3):e0151893.

20. Gotoda T, Takano C, Kusano C, Suzuki S, Ikehara H, Hayakawa S, Andoh A. Gut microbiome can be restored without adverse events after Helicobacter pylori eradication therapy in teenagers. Helicobacter. 2018;23(6):e12541.

21. Liou JM, Chen CC, Chang CM, Fang YJ, Bair MJ, Chen PY, Chang CY, Hsu YC, Chen MJ, Chen CC, et al. Long-term changes of gut microbiota, antibiotic resistance, and metabolic parameters after Helicobacter pylori eradication: a multicentre, open-label, randomised trial. Lancet Infect Dis. 2019;19(10):1109-20.

22. Dash NR, Khoder G, Nada AM, Al Bataineh MT. Exploring the impact of Helicobacter pylori on gut microbiome composition. PloS one. 2019;14(6):e0218274.

23. Bourke B, Ceponis P, Chiba N, Czinn S, Ferraro R, Fischbach L, Gold B, Hyunh H, Jacobson K, Jones $\mathrm{NL}$, et al: Canadian Helicobacter Study Group Consensus Conference: Update on the approach to Helicobacter pylori infection in children and adolescents-an evidence-based evaluation. Can J Gastroenterol 2005, 19(7):399-408.

24. Jones NL, Koletzko S, Goodman K, Bontems P, Cadranel S, Casswall T, Czinn S, Gold BD, Guarner J, Elitsur Y, et al. Joint ESPGHAN/NASPGHAN Guidelines for the Management of Helicobacter pylori in Children and Adolescents (Update 2016). J Pediatr Gastroenterol Nutr. 2017;64(6):991-1003.

25. Koletzko S, Jones NL, Goodman KJ, Gold B, Rowland M, Cadranel S, Chong S, Colletti RB, Casswall T, Elitsur Y, et al. Evidence-based guidelines from ESPGHAN and NASPGHAN for Helicobacter pylori infection in children. J Pediatr Gastroenterol Nutr. 2011;53(2):230-43.

26. Asaka M, Kato M, Takahashi S, Fukuda Y, Sugiyama T, Ota H, Uemura N, Murakami K, Satoh K, Sugano K. Guidelines for the management of Helicobacter pylori infection in Japan: 2009 revised edition. Helicobacter. 2010;15(1):1-20.

\section{Figures}


Figure 1

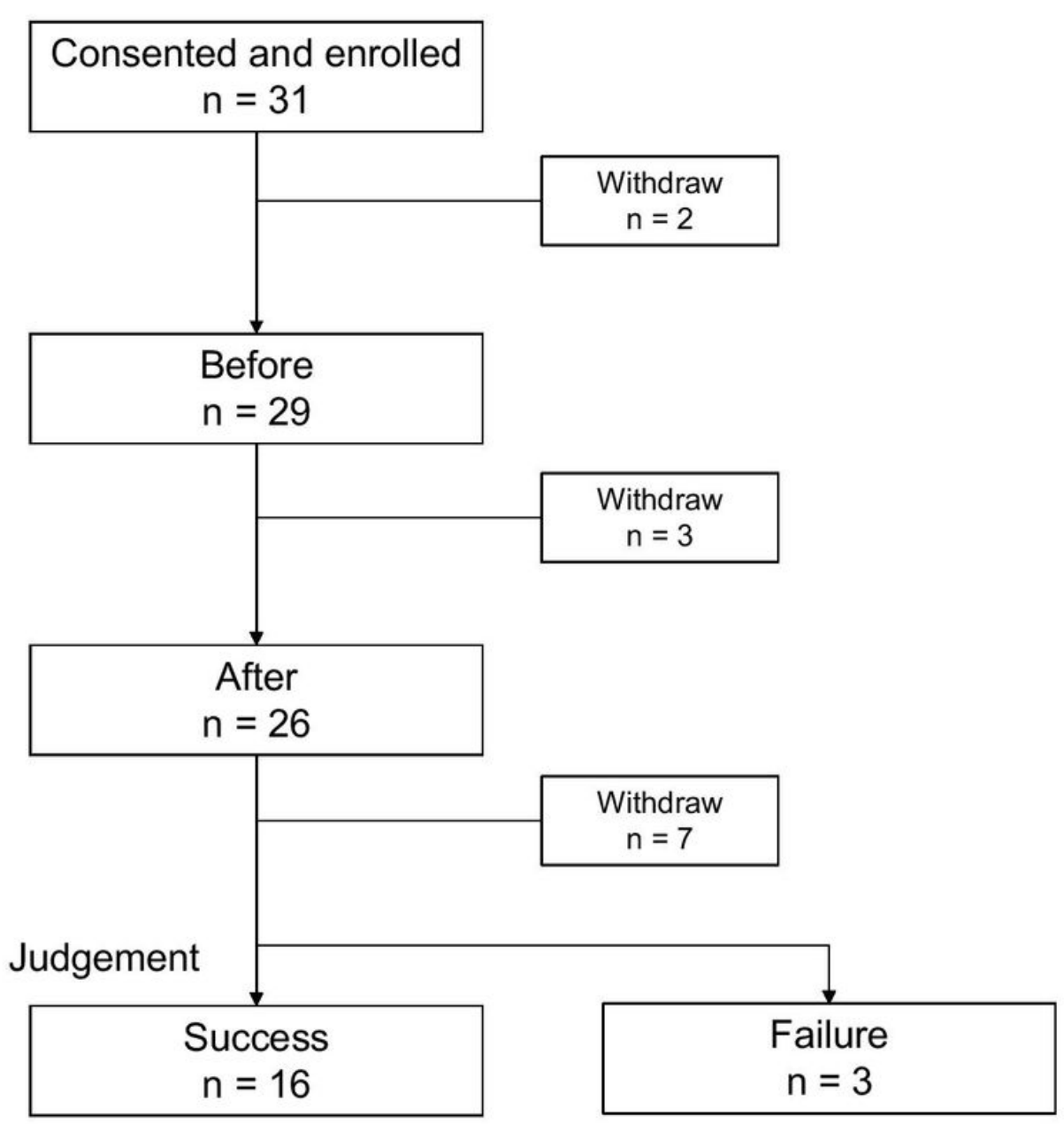

Figure 1

Flow chart of patient enrolment. 


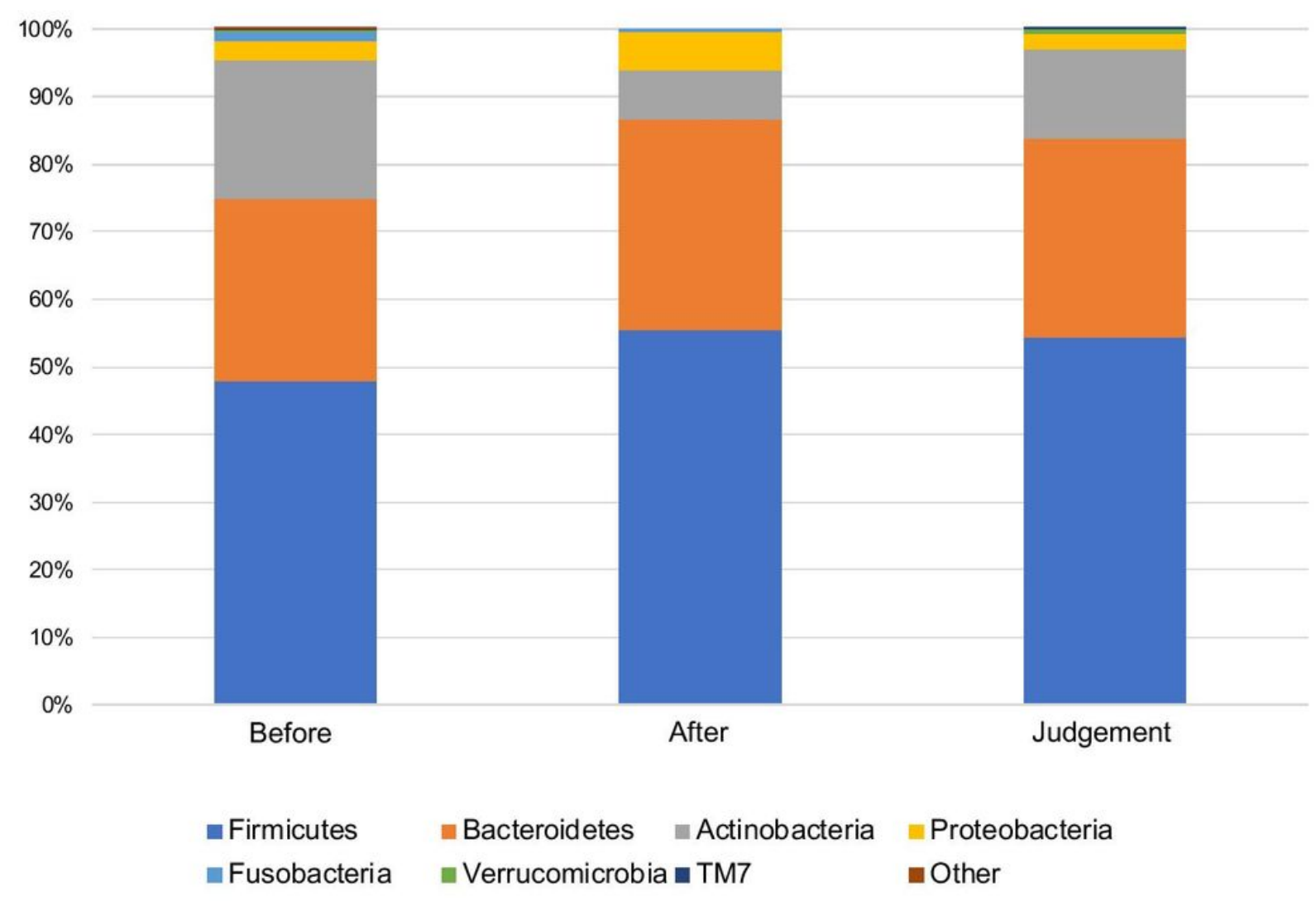

\section{Figure 2}

Relative abundance of the intestinal microbiota at the phyla at three time points: before eradication therapy, after eradication therapy, and at the time of eradication therapy judgment. 


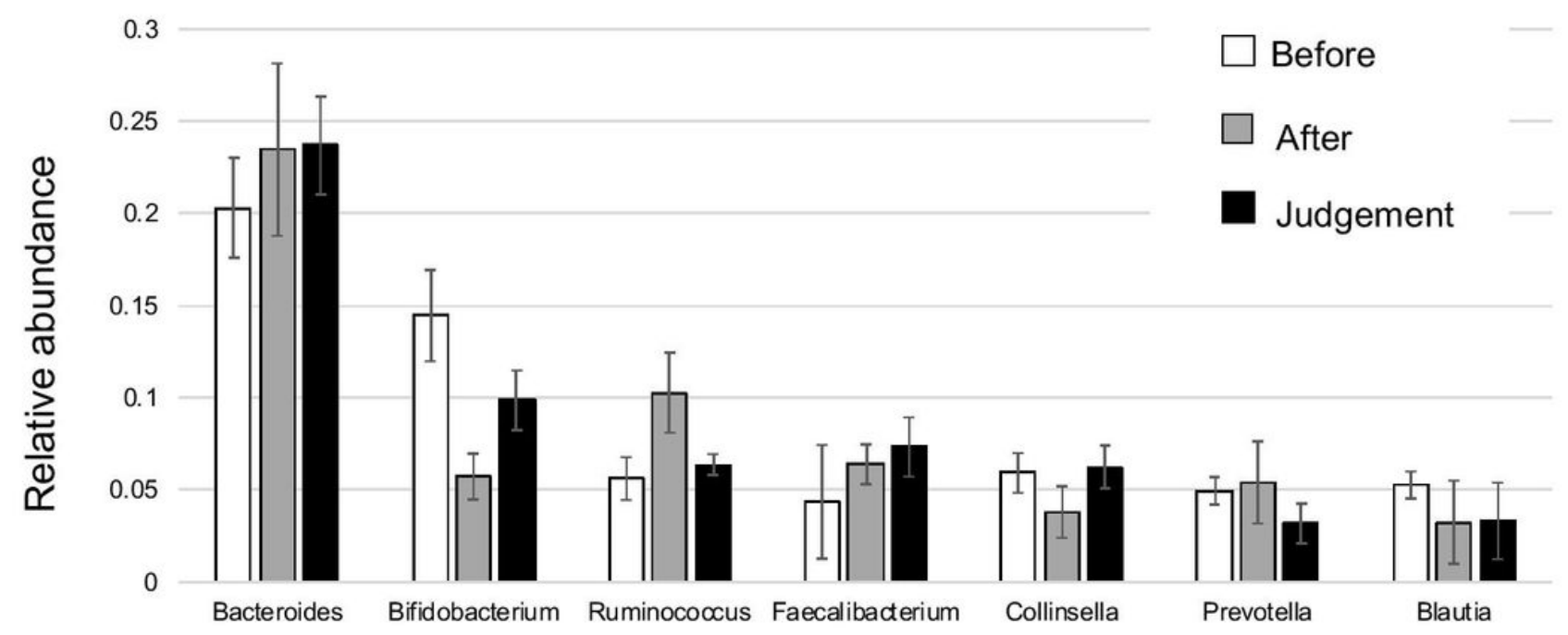

Figure 3

Relative abundance of the intestinal microbiota larger than $3 \%$ at three time points: before eradication therapy, after eradication therapy, and at the time of eradication therapy judgment. 
(A)

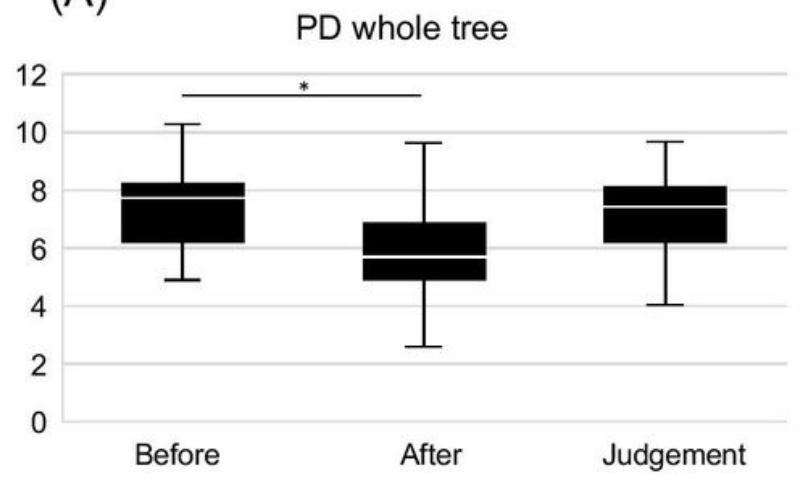

(C)

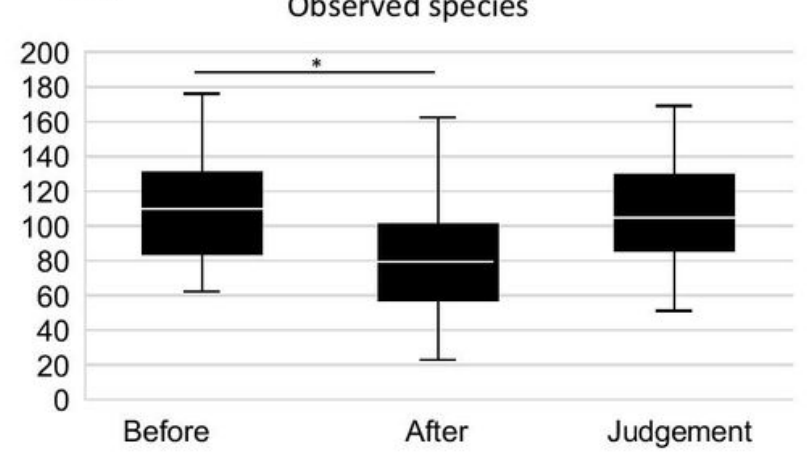

(B)

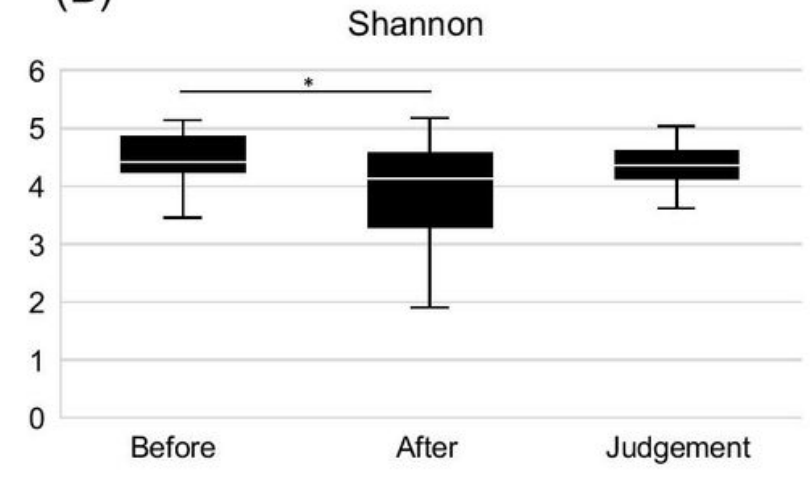

(D)

Chao1

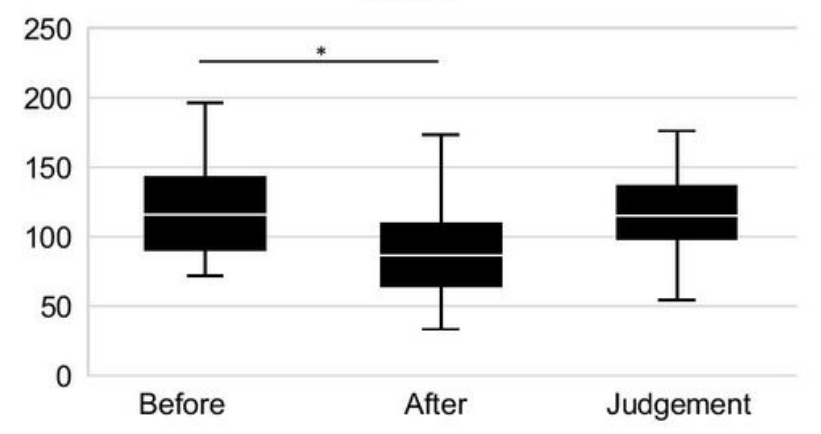

\section{Figure 4}

Boxplot depicting a-diversity before eradication therapy, after eradication therapy, and at the time of eradication judgment. (A) PD whole tree, (B) Shannon index, (C) Observed OTUs, and (D) PD whole tree. Comparison before and after eradication therapy ( $P D$ whole tree, $P=0.003$; Shannon index, $P=0.023$; observed OTUs, $\mathrm{P}=0.003$; and Chao1, $\mathrm{P}=0.001$ ). Comparison between before eradication therapy and at the time of eradication therapy judgment (PD whole tree, $P=1.000$; Shannon index, $P=1.000$; observed OTUs, $\mathrm{P}=1.000$; and Chao1, $\mathrm{P}=1.000)$. Comparison between after eradication treatment and at the time of eradication therapy judgment $(P D$ whole tree, $\mathrm{P}=0.063$; Shannon index, $\mathrm{P}=0.492$; observed OTUs, $\mathrm{P}=$ 0.106; and Chao1, $\mathrm{P}=0.061$ ). 
(A)

Weight UniFrac distances

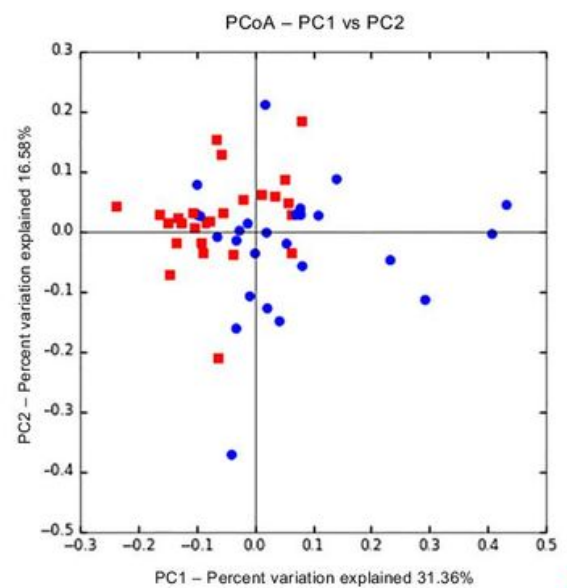

(C)

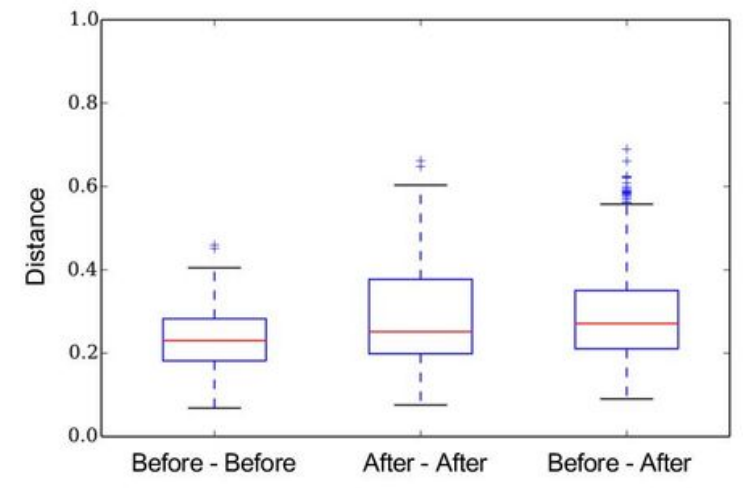

(B)

Unweight UniFrac distances

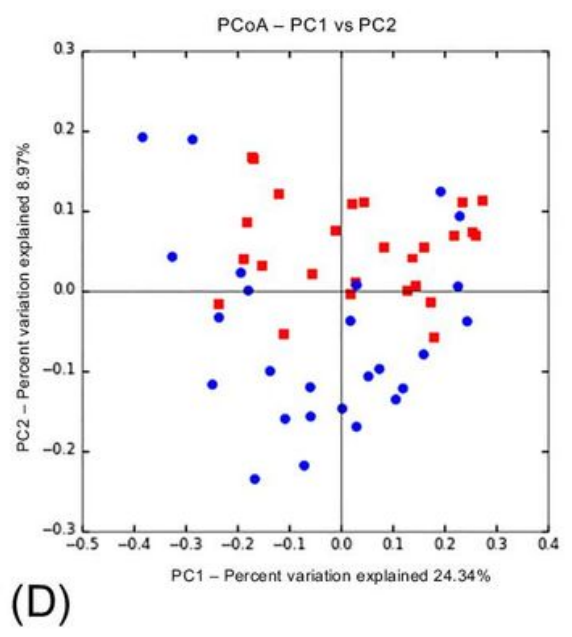

\section{Figure 5}

Principal coordinates analysis revealed clustered communities of gut microbiota after eradication in the before and after eradication therapy groups. (A) Weighted UniFrac distances, (B) Unweighted UniFrac distances. Bar diagram showing the Mean UniFrac distances for Before-Before, After-After, and BeforeAfter participants. (C) Weighted UniFrac distances (Before-Before vs. Before-After, $P=0.03$; After-After vs. Before-After, $P=1.00$ ), (D) Unweighted UniFrac distances (Before-Before vs. Before-After, $P=0.03$; After-After vs. Before-After, $P=0.98)$. 
(A)

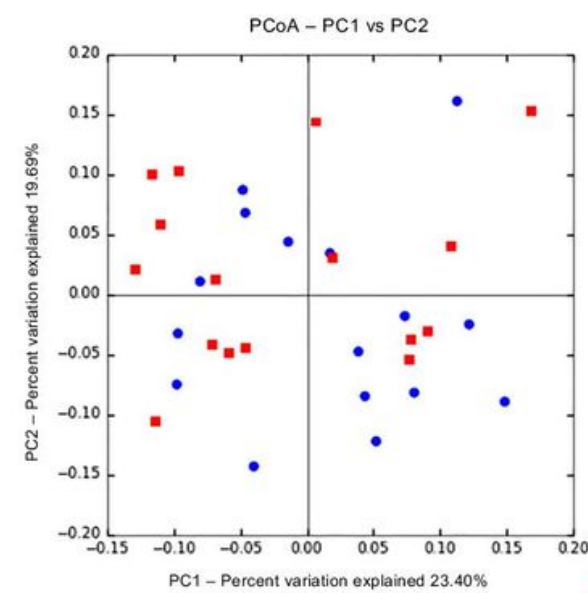

(C)

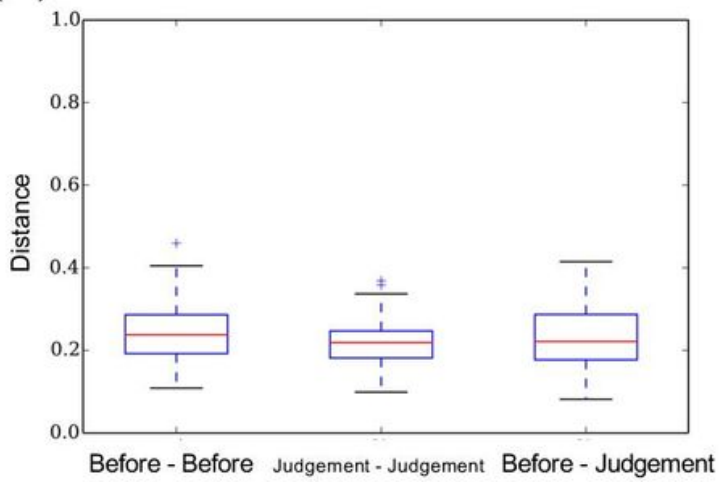

(B) Unweight UniFrac distances

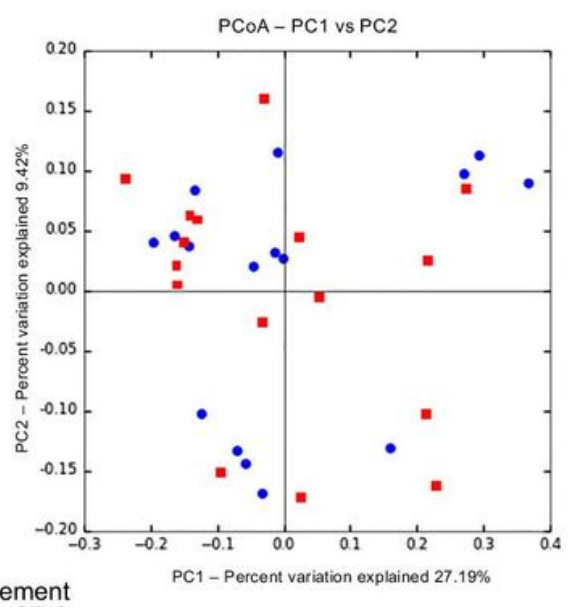

(D)

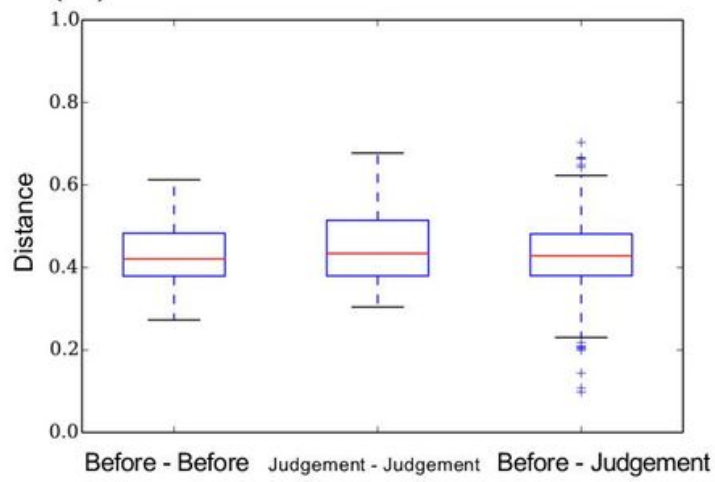

\section{Figure 6}

Principal coordinates analysis revealed clustered communities of gut microbiota after eradication in the before and after eradication therapy groups. (A) Weighted UniFrac distances, (B) Unweighted UniFrac distances. Bar diagram showing the Mean UniFrac distances for Before-Before, Judgment-Judgment, and Before-Judgment participants. (C) Weighted UniFrac distances (Before-Before vs. Before Judgment, $P=1.00$; Judgment-Judgment vs. Before-Judgment, $P=1.00)$, (D) Unweighted UniFrac distances (Before -Before vs. Before -Judgment, $P=1.00$; Judgment -Judgment vs. Before-Judgment, $P=0.87)$. 